\title{
SOL RF physics modelling in Europe, in support of ICRF experiments
}

Laurent Colas ${ }^{1, *}$, LingFeng Lu ${ }^{1,2,3, \dagger}$, Jonathan Jacquot ${ }^{4}$, Wouter Tierens ${ }^{4}$, Alena Křivská ${ }^{5}$, Stéphane Heuraux $^{2}$, Eric Faudot $^{2}$, Patrick Tamain ${ }^{1}$, Bruno Després ${ }^{6}$, Dirk Van Eester ${ }^{5}$, Kristel Crombé ${ }^{3,5}$, Fabrice Louche $^{5}$, Julien Hillairet ${ }^{1}$, Walid Helou ${ }^{1}$, and Marc Goniche ${ }^{1}$

${ }^{1}$ CEA, IRFM, F-13108 Saint Paul Lez Durance, France.

${ }^{2}$ Institut Jean Lamour, UMR 7198, CNRS-University of Lorraine, F-54506 Vandoeuvre Cedex, France.

${ }^{3}$ Department of Applied Physics, Ghent University, Belgium

${ }^{4}$ Max-Planck-Institut für Plasmaphysik, Garching, Germany.

${ }^{5}$ LPP-ERM-KMS, TEC partner, Brussels, Belgium.

${ }^{6}$ Laboratoire Jacques Louis Lions, UPMC-Paris VI, CNRS UMR 7598, Paris, France.

$\dagger$ present address: TianQin Research Center for Gravitational Physics, School of Physics and Astronomy, Sun Yat-Sen University, Zhuhai 519082, P. R. China

\begin{abstract}
A European project was undertaken to improve the available SOL ICRF physics simulation tools and confront them with measurements. This paper first reviews code upgrades within the project. Using the multi-physics finite element solver COMSOL, the SSWICH code couples RF full-wave propagation with DC plasma biasing over "antenna-scale" 2D (toroidal/radial) domains, via non-linear RF and DC sheath boundary conditions (SBCs) applied at shaped plasma-facing boundaries. For the different modules and associated SBCs, more elaborate basic research in RF-sheath physics, SOL turbulent transport and applied mathematics, generally over smaller spatial scales, guides code improvement. The available simulation tools were applied to interpret experimental observations on various tokamaks. We focus on robust qualitative results common to several devices: the spatial distribution of RF-induced DC bias; left-right asymmetries over strap power unbalance; parametric dependence and antenna electrical tuning; DC SOL biasing far from the antennas, and RF-induced density modifications. From these results we try to identify the relevant physical ingredients necessary to reproduce the measurements, e.g. accurate radiated field maps from 3D antenna codes, spatial proximity effects from wave evanescence in the near RF field, or DC current transport. Pending issues towards quantitative predictions are also outlined.
\end{abstract}

\section{ICRF antennas as active Plasma- Facing Components}

The phased strap arrays used to launch Ion Cyclotron Range of Frequency (ICRF, 30-80MHz) waves into magnetic fusion devices poorly radiate in vacuum: efficient coupling of the fast wave to the main plasma necessitates minimizing the distance from the straps to a critical peripheral density: the $R$-cutoff layer. In the Scrape-Off Layer (SOL) the antennas then behave as Plasma-facing Components (PFCs), subject to PlasmaSurface Interactions (PSI). As RF-field-emitting structures, ICRF antennas are active PFCs able to create RF-specific PSI and to modify locally their environment: enhanced ion energies, heat loads, erosion and density modifications have been observed locally. These phenomena are critical in the prospect of long-pulse machines with high- $Z$ plasma-facing materials. Predicting the magnitude and spatial location of these processes, in relation with plasma parameters, launcher design and electrical settings, remains challenging. So far, realistic tokamak predictions relied on very simple models of oscillating double probes. A European project was undertaken to improve the available SOL RF physics simulation tools and confront them with measurements. This paper first reviews code improvements and associated basic research within the project. The available simulation tools are then applied to interpret experimental observations on various machines. From these results we exhibit qualitative properties common to the various devices, identify relevant physical ingredients necessary to reproduce the measurements, as well as pending issues towards quantitative predictions.

\section{Improvements in RF+DC sheaths simulations, associated basic research}

The intense time-harmonic RF electric fields $\mathbf{E}$ emitted in the SOL at frequency $\omega_{0}$ are generally suspected to cause RF oscillations $V_{R F} \exp \left(-\mathrm{i} \omega_{0} t\right)$ of the sheath voltage at plasma-wall interfaces. Non-linear rectification of these oscillations then produces a Direct-Current (DC) self-biasing of the SOL plasma. Ion acceleration across the larger DC potential $V_{D C}$ is suspected to enhance the PSI locally. Sheaths also modify the RF wave reflection at material boundaries, in a way depending on $V_{D C}$. Our simulations over spatial scales comparable to antenna dimensions couple simple models of RF wave

\footnotetext{
Corresponding author: laurent.colas@cea.fr
} 
propagation for $\mathbf{E}$ and DC SOL biasing for $V_{D C}$ via nonlinear sheath electrical properties. In Europe, these are implemented in the Self-consistent Sheaths and Waves for Ion Cyclotron Heating (SSWICH) code [1], [2], [3], using the Newton-Raphson scheme in the multiphysics solver COMSOL. As an intermediate step the code also computes the complex oscillating voltages $V_{R F}$ at sheath boundaries. Rectification likely generates harmonics of the wave frequency $\omega_{0}$ but these are neglected in our modelling. We examine below the different modules successively. In several key areas, more elaborate basic research in physics and applied mathematics, generally over smaller spatial scales, guides code improvement.

\subsection{RF and DC sheath boundary conditions.}

In "antenna-scale" modelling, limited spatial resolution presently imposes treating the sheaths as non-linear sheath boundary conditions (SBCs) at plasma/sheath interfaces, applied at plasma-facing material boundaries. SSWICH presently incorporates simple RF and DC SBCs.

DC SBCs in the DC biasing module reflect the rectifying properties of the sheaths. Current-voltage $(j / V)$ characteristics of sheaths are generally non-linear. Consider for simplicity the case of a static sheath

$$
j=j^{+}\left[1-\exp \left[-e\left(V-V_{f}\right) / k T_{e}\right]\right.
$$

With $T_{e}$ the electron temperature, $V_{f}$ the floating potential in the absence of RF sheaths, and $j^{+}$the ion saturation current. When the confinement magnetic field $\mathbf{B}_{\mathbf{0}}$ is tilted by angle $\alpha$ with respect to the wall, $j+$ is supposed to scale as $\sin (\alpha)$ [3]. Due to the non-linearity in (1), adding a sinusoidal RF oscillations $V_{R F}$ of $V$ on top of a DC component $V_{D C}$, and time averaging $j(t)$ shifts the $j / V$ characteristics

$$
j_{D C}=j^{+}\left[1-\exp \left[-e\left(V_{D C}-V_{b}-V_{f}\right) / k T_{e}\right]\right.
$$

Oscillating sheaths behave as if the (grounded) wall was locally biased to a DC voltage $V_{b}$ expressed as

$$
V_{b}=k T_{e} \log \left[I_{0}\left(e\left|V_{R F} / k T_{e}\right|\right)\right] / e
$$

With $I_{0}$ the modified Bessel function. DC SBCs (2) therefore couple the DC biasing to RF wave-propagation via $V_{R F}$. Conversely the RF SBCs couple the wave propagation to the $\mathrm{DC}$ bias. At the RF timescale, RF sheaths are often treated as thin dielectric layers of width $\delta$ between the wall and the quasi-neutral plasma. The associated RF SBC was first formulated in [4] and reads

$$
V_{R F}=D_{n} \delta / \varepsilon_{\mathrm{sh}}
$$

where $D_{n}$ is the component of the electric displacement normal to the boundary, $\varepsilon_{\mathrm{sh}}$ is a dielectric constant of order 1 and the sheath width $\delta$ depends on the DC sheath voltage $V_{D C}$ via the Child-Langmuir law.

$$
\delta \sim \lambda_{D e}\left(e V_{D C} / k T_{e}\right)^{3 / 4}
$$

where $\lambda_{D e}$ is the electron Debye length at the entrance of the Debye sheath. Consistent with the electrostatic sheath approximation, the RF field $\mathbf{E}_{\mathbf{t}}$ tangent to the sheath/plasma interface is not null

$$
\mathbf{E}_{\mathbf{t}}=-\nabla_{\mathbf{t}} V_{R F}
$$

The electrostatic approximation also imposes that

$$
B_{n}=-\mathrm{i}(\nabla \times \mathbf{E})_{n} / \omega_{0}=\mathrm{i} \nabla \times\left(\nabla_{\mathbf{t}} V_{R F}\right) / \omega_{0}=0
$$

To start the iterations between RF and DC modules, RF wave propagation is first run using asymptotic RF SBCs valid for large sheath widths and independent of $V_{D C}$. Together with relation (7), the general form of the asymptotic RF SBC reads [1], [5].

$$
D_{n}=0
$$

Since their first formulation, SBCs have been improved using more basic models of spatially-resolved magnetized RF sheaths over scales much smaller than antenna dimensions [6], [7]. The structure of SSWICH is modular and can incorporate these improvements progressively. Several basic models were tested in Europe. Paper [8] investigated the linear reflection of cold plane waves on a flat boundary across a prescribed time-independent 1D density profile characteristic of a sheath. Such plane waves exist with Fast Wave or Slow Wave (SW) polarization. As already shown by [9] such incoming waves are generally depolarized upon reflection. The process is described via a $2 \times 2$ matrix relation between incident and outgoing Fast and SW, depending on plasma profiles, $\alpha$ and tangent wavevector. The parametric sensitivity of the matrix components was stressed in [8]. In [10] the above model was made more sophisticated: the intense RF fields change the DC density profile via ponderomotive forces, in addition to the usual electric and pressure forces in the DC sheath. This in turn modifies the wave propagation. Selfconsistent simulations were performed. Similar to [11], kinetic $1 \mathrm{~d} 3 \mathrm{v}$ PIC simulations of an oscillating double Langmuir probe are used to refine the RF impedance (4), $j_{D C} / V_{D C}$ characteristics (2) (3), and sheath widths (5) of magnetized RF sheaths, as a function of $\alpha$, relevant frequencies $\left(\omega_{0}\right.$, plasma frequency $\omega_{\text {pi }}$, cyclotron frequency $\Omega_{\mathrm{ci}}, \ldots$ ) and temperatures. First runs suggest that $V_{b}$ in equation (3) should depend on $\alpha$ : it tends to vanish below the Chodura angle where DC sheaths disappear: $\sin \left(\alpha_{c}\right)=\left[2 \pi\left(1+T_{i} / T_{e}\right) m_{e} / m_{i}\right]^{1 / 2}$. Other valuable output includes the time-averaged ion energy distribution at the wall as well as higher harmonics of the wave frequency $\omega_{0}$.

\subsection{RF wave propagation module for tokamaks}

RF wave propagation is solved using the Finite Element Method (FEM) to allow versatile wall shapes and plasma in contact with material boundaries for sheath excitation. The original SSWICH-SW version solves for the scalar field $E_{/ /}$representing the time harmonic cold SW [2] 


$$
\varepsilon_{/ /} \Delta_{/ /} E_{/ /}+\varepsilon_{\perp} \Delta_{\perp} E_{/ /}+k_{0}^{2} \varepsilon_{/ /} \varepsilon_{\perp}=0
$$

With $k_{0}=\omega_{0} / c$ and $\left(\varepsilon_{/ /}, \varepsilon_{\perp}\right)$ the cold-plasma dielectric constants [12]. Walls are either parallel or normal to $\mathbf{B}_{\mathbf{0}}$. Excitation is provided by a map of $E_{/ /}$from an external antenna code, and prescribed at the antenna mouth (see Figure 1). Both fully coupled versions and asymptotic RF-SBCs exist, in a 2D (radial, toroidal) plane.

Within the project SSWICH was upgraded to fullwave polarization to solve the vectorial wave equation

$$
\nabla \times \nabla \times \mathbf{E}-k_{0}^{2} \mathbf{E E}=0
$$

excited either by straps or vectorial input field maps (see Figure 1). A 2D version was tested in [3] including shaped walls and magnetized RF sheaths. Eq. (6) was used to integrate $V_{R F}$ along the curved 1D boundary, assuming $V_{R F}=0$ far from the antenna. A standalone 3D version of SSWICH-FW with metallic BCs and embedded antenna was developed as RAPLICASOL [13]. It is presently used as an antenna code in [14], [15]. An iterative solver (GM-RES) reduces the huge RAM requirements of $3 \mathrm{D}$ computations.

Figure 1 sketches the simulation domain for the RF module of SSWICH-FW. Focus is put on the antenna vicinity at the Low Field Side (LFS) of a straightened tokamak and does not cover the whole radial and toroidal extension of the vessel. It cannot investigate RFsheaths likely present at the high-field side (HFS). The magnetic connection lengths at LFS are also reduced compared to reality. In order to fairly reproduce the power coupling in presence of inhomogeneous plasma, the radial extent of the simulation domain needs to include all the relevant $R$-cutoffs in the radiated $k_{/ /}$ spectrum.

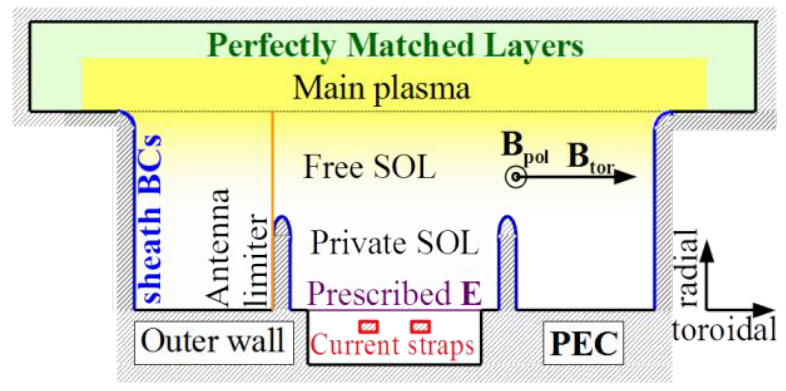

Fig. 1. Sketch of 2D (toroidal/radial) simulation domain for the RF wave module of SSWICH-FW in tokamaks (not to scale). Confinement magnetic field $\mathbf{B}_{\mathbf{0}}$ includes in-plane $\left(\mathrm{B}_{\text {tor }}\right)$ and out-of-plane $\left(\mathrm{B}_{\mathrm{pol}}\right)$ components (see Figure 3 ). Colour intensities are indicative of plasma local density. RF waves are excited either by prescribed RF currents on poloidal straps or prescribed RF electric field tangent to the magenta boundary (cut through "antenna aperture" on Figure 3). RF SBCs are implemented at blue boundaries. Orange line: cut through "reference plane" on Figure 3.

At the inner part of the domain, SSWICH-FW and RAPLICASOL emulate radiation to infinity using Perfectly Matched Layers (PMLs). Inside PMLs, spatial coordinates are stretched into the complex plane, turning propagative waves into evanescent ones. The only restriction is to avoid coexisting forward and backward waves at PML location. PMLs are implemented as lossy inhomogeneous materials with artificial dielectric and magnetic tensors depending on plasma parameters, the stretching function and stretched coordinates. For gyrotropic media like cold plasmas, PMLs were formulated in slab [17] and cylindrical geometries [13].

The FEM allows versatile spatial distributions of the plasma parameters. In the radial direction this includes realistic density profiles from tokamak measurements. Inhomogeneity can also be implemented in the other directions. Paper [2] defined a private SOL surrounded by the two antenna limiters on figure 1. References [16], [18] investigated waves in presence of RF-induced density modifications.

As one such application, the impact of a tenuous plasma inside a realistic ICRF antenna box was explored in [20], using RAPLICASOL in 2D. The tenuous plasma introduces the Lower-Hybrid $(\mathrm{LH})$ resonance in the box when $\varepsilon_{\perp}=0$. The SW excited parasiticallybecomes propagative at densities below LH resonance. Local RF fields in this region evolved upon mesh refinement. This is attributed to the short wavelength of the SW, combined with the box geometry, a domain without volume losses closed by metallic walls on three sides, and coupled to the straps via the LH resonance. This complicates near field studies in the box. Yet, the radiated power was found independent of the mesh: the sloshing power carried by the SW is transferred to the fast wave. The radiated power spectrum was weakly dependent on the density in the box, except for low $k_{/ /}$ components, whose $R$-cutoff moved closer to the straps in presence of tenuous plasma.

Crossing the cold LH resonance causes specific numerical issues for the fast wave in perpendicular propagation: the radial electric field exhibits a singularity there, causing finite power damping in the collisionless limit [21]. Regularization relies frequently on artificial friction, i.e. an imaginary part $v$ added to $\omega_{0}$, e.g. in [13]. Numerical tests however show that a subtle trade-off between the choices of $v$ and mesh size is necessary to recover the collisionless limit $v \rightarrow 0^{+}$. An alternative formulation was proposed in [22], [23]: at the resonance position, the wave equation is replaced by an integral relation involving only the non-singular field components, as well as collisionless "manufactured solutions". Numerical tests show the good behaviour of 1D FEM using this scheme against analytical solutions of the Budden problem. Extension to 2D is under study.

\subsection{Full-wave RF wave propagation in ALINE}

Europe hosts two RF-sheath testbeds: IShTAR [24] and ALINE [25], [26]. ALINE is a cylindrical device (length $1 \mathrm{~m} \times$ diameter $30 \mathrm{~cm}$ ) producing partially ionized $\mathrm{Ar}$ and He plasmas with longitudinal magnetic fields up to $0.1 \mathrm{~T}$ and electron densities in the range of $10^{16} \mathrm{~m}^{-3}$. Plasma and waves (frequencies $10 \mathrm{kHz}-250 \mathrm{MHz}$ ) are produced by a cylindrical RF electrode located at the centre of the vessel. Waves are damped in the plasma volume by 
collisions. The ALINE plasma can be mapped in 3D by a RF-compensated moveable Langmuir probe.

SSWICH was recently adapted to ALINE. The simulation domain for this device, sketched on figure 2, features a $2 \mathrm{D}$ cut across the closed vacuum vessel, filled with cold collisional magnetized plasma. RF excitation is provided by imposing an oscillating voltage between the vessel body and the coaxial feeder of the RF electrode, surrounded by a ceramic tube. Asymptotic RF SBCs are implemented at vessel boundaries normal to $\mathbf{B}_{\mathbf{0}}$ while perfect conductors are assumed on walls parallel to $\mathbf{B}_{\mathbf{0}}$

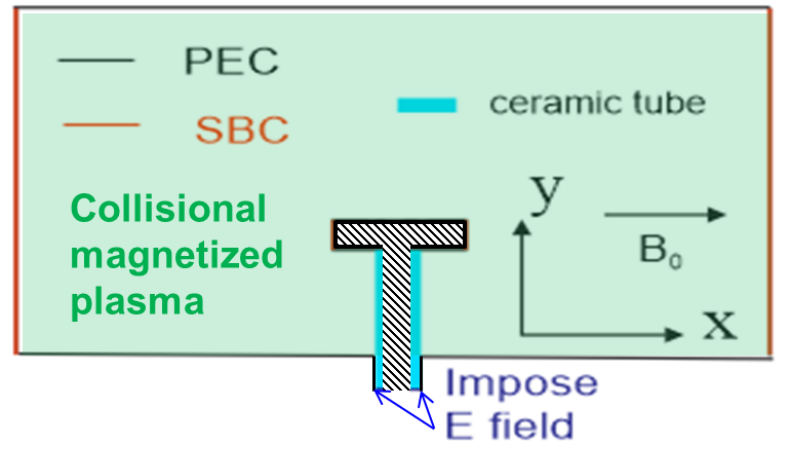

Fig. 2. 2D (axial/longtitudinal) simulation domain for the RF wave module of SSWICH-FW in ALINE. Not to scale.

\subsection{DC plasma biasing module of SSWICH}

The DC plasma biasing module of SSWICH solves the conservation of DC charge over the SOL.

$$
\operatorname{div}\left(\mathbf{j}_{\mathbf{D C}}\right)=0
$$

At the separatrix the plasma is supposed to be floating. DC SBCs implement $j_{D C} / V_{D C}$ characteristics (2) with artificial DC wall bias $V_{b}$ (3) driving enhanced DC plasma potentials $V_{D C}$. In the volume the conduction is supposed to follow a highly anisotropic Ohm's law

$$
\mathbf{j}_{\mathbf{D C}}=-\boldsymbol{\sigma}_{\mathbf{D C}} \nabla \mathbf{V}_{\mathbf{D C}}
$$

In the parallel direction the Spitzer DC conductivity is taken, while a phenomenological conductivity is introduced transverse to $\mathbf{B}_{\mathbf{0}}$. In references [2] and [15] typical quantitative values in the range $\sigma_{\perp \mathrm{DC}} \sim 10^{-6} \sigma_{/ / \mathrm{DC}}$ in the antenna private SOL and $\sigma_{\perp \mathrm{DC}} \sim 10^{-8} \sigma_{/ / \mathrm{DC}}$ in the main SOL were found to reasonably fit the measurements. Relation (12) is strictly valid only for charged particles colliding with neutrals. This process is however quite weak in the tokamak SOL. The transverse transport of DC current is more likely turbulent, but SSWICH cannot afford modelling it. Therefore $\sigma_{\perp D C}$ is a loosely constrained parameter.

The above DC current transport process was assessed numerically against 3D SOL turbulence simulations using the TOKAM3X fluid code [27]. As a proxy to the self-biasing by sheath rectification, a local DC bias was imposed on material boundaries protruding into the outer half of the SOL, similar to the antenna side limiters on Figure 1. From these biased wall elements, the DC potential spread along connected magnetic flux tubes over long distances, with parallel attenuation of the peaks and broadening to adjacent field lines. An Ohm's law like (12) was found inadequate to describe the local transverse conduction. No linear relation exists between local DC currents and DC electric fields. Besides, the simulated conduction was different in the radial and poloidal directions. The local shear of the DC electric field $\mathbf{E}_{\mathbf{D C}}=-\nabla_{\perp} V_{D C}$ in the biased region likely plays a role in the turbulent conduction. Deeper studies will however be needed to determine a more accurate mean-field description of transverse anomalous charge transport.

\subsection{Brief panorama of antenna-scale RF-sheath modelling}

SSWICH is a unique tool in Europe to model RFsheath effects over antenna-scale spatial domains in tokamaks. Similar tools however exist worldwide. Their main characteristics are compared in table 1. Although all these tools make use of RF-SBCs, each one features specificities. Vsim [28] uses the Finite Difference Time Domain (FDTD) method. Although this is not yet implemented, it can potentially handle altogether all the harmonics of the wave frequency and the full nonlinearity of the initial RF-sheath problem, as well as ponderomotive effects. rfSOL [5] models an entire poloidal cross-section of the machine. It was used to investigate qualitatively RF-sheaths at the HFS walls, due to residual fast waves crossing the plasma core [29]. The DC plasma biasing module allowing DC current transport is a specificity of SSWICH. Its necessity will be further discussed in the context of tokamak experiments. All alternative codes implicitly assume that the sheaths float over time-scales much longer than the RF period, so that no DC current crosses them. The local DC potential $V_{D C}$ at one sheath then depends only on the local $V_{R F}$. It is independent of the sheaths at the opposite extremity of the same field line or on neighbouring flux tubes.

Table 1. Existing "antenna-scale" RF models with RF-sheath boundary conditions for tokamaks

\begin{tabular}{|c|c|c|c|c|c|}
\hline & Method & Dimensions & RF module & DC module & Excitation \\
\hline Vsim [28] & FDTD & $3 \mathrm{D}$ & \multicolumn{2}{|c|}{$\begin{array}{c}\text { Time-resolved } \\
\text { floating sheaths }\end{array}$} & $\begin{array}{c}\text { CAD-based } \\
\text { antenna model }\end{array}$ \\
\hline rfSOL [5] & FEM & $\begin{array}{c}\text { 2D cross-section } \\
\text { (radial/poloidal) }\end{array}$ & Full Wave & Floating sheaths & Current sheet \\
\hline SSWICH & FEM & $\begin{array}{c}\text { Multi-2D, LFS } \\
\text { (radial/toroidal) }\end{array}$ & $\begin{array}{c}\text { Slow Wave or } \\
\text { Full Wave }\end{array}$ & $\begin{array}{c}\text { DC currents } \\
\text { (Ohm's law) }\end{array}$ & $\begin{array}{c}\text { Straps/realistic } \\
\text { field maps }\end{array}$ \\
\hline
\end{tabular}




\section{Application of SSWICH to qualitatively interpret tokamak experiments.}

The SSWICH-SW code was applied for the first time in 2012 to interpret observations on Tore Supra (TS) [2]. Predictions were also made for WEST and ITER [30]. The project revisited the TS simulations with the upgraded SSWICH-FW tool [3], and extended the tokamak database to ASDEX upgrade (AUG) 2-strap and 3-strap antennas [14], [15] as well as JET ITER-like Antenna (ILA) [31].

Mostly run was the SSWICH-SW version using asymptotic RF-SBCs (7) and (8), with no attempt at selfconsistency. The fully-coupled SSWICH-SW version was run in a few TS cases, showing that the asymptotic approximation, corresponding to the first turn around the self-consistent loop, was a fair approximation of the final result in these cases [2]. In [3] the TS simulations were also revisited using the SSWICH-FW code with asymptotic RF-SBCs. Specific effects related to the Fast Wave were investigated. In all cases a multi-2D procedure was followed and is sketched on Figure 3 for SSWICH-FW: using 2D (toroidal/poloidal) input field maps from 3D antenna codes with plasma load but without sheaths (either TOPICA [32] or RAPLICASOL [13]), independent 2D (radial/toroidal) simulations were performed at each altitude along the antenna mouth, assuming negligible poloidal gradients. For SSWICHSW simulations, this multi-2D procedure was assessed in [33]: it is valid as long as the input RF field maps do not exhibit small-scale poloidal structures below the electron skin depth $c / \omega_{p e}$. The multi-2D approach will be further discussed below for Fast Wave propagation.

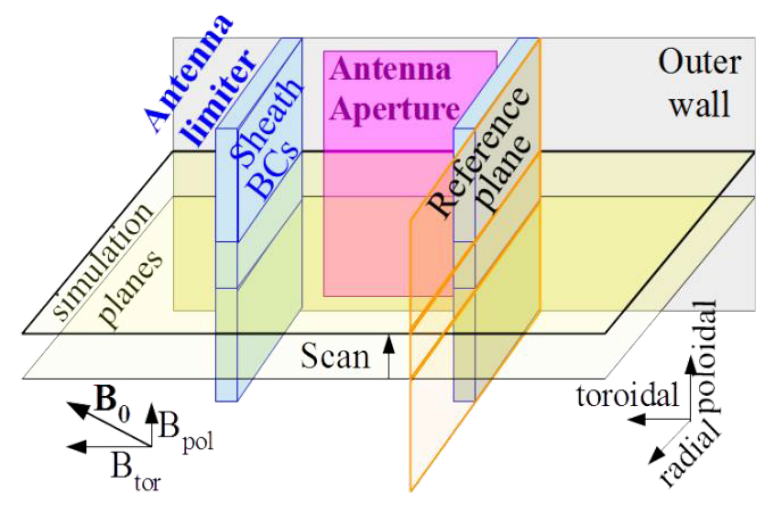

Fig. 3. 3D sketch of multi-2D simulation procedure with SSWICH-FW code excited using antenna codes. Independent 2D runs are successively performed in each (toroidal/radial) simulation plane, detailed on Figure 1 and visualized here from plasma side. They take as excitation poloidal cuts through a 2D (toroidal/poloidal) input field map prescribed at antenna aperture. From the simulation output, $V_{D C}$ is mapped in section 3.1 in the orange (radial/poloidal) reference plane.

Quantitative sensitivity of the simulations on loosely constrained parameters is frequently reported [20], [15], [2]. We concentrate here on relative comparisons and robust qualitative results reproducible over many runs, on several machines, in relation with experimental observations.

\section{1. $V_{D C}$ spatial structure near antenna limiters.}

The RF-induced PSI does not affect the SOL uniformly, but rather concerns a limited number of flux tubes. 2D (radial/poloidal) maps of the DC plasma potential $V_{D C}$ were simulated on the external face of antenna side limiters (reference plane on Figure 3) for active TS antennas [2], [3], AUG 2-strap and 3-strap antennas [15], [14]. All these maps exhibit similar spatial structures. $V_{D C}$ in the free SOL is peaked radially at the leading edge of the limiters. A 2-hump poloidal variation appears, with local maxima near the poloidal extremities of the antenna box, and local minima near the equatorial plane. Such spatial structure was observed experimentally on TS [34] and AUG [35] for ion parallel energies. A 2-hump poloidal structure was also present for heat loads along TS antenna limiters [36] and W sputtering yield on AUG [37]. A 2-hump structure was also visualized on the LAPD testbed [38].

The JET ILA consists of two strap arrays on top of each other that can be powered independently. When the upper ILA runs in dipole, numerical $V_{D C}$ peaks are present near the poloidal extremities of the upper ILA [31]. The peaks move to the poloidal extremities of the lower array when it is powered, while smaller peaks exist at the poloidal ends of the whole box when the full array radiates. Correlation of the poloidal structure with BeII line intensity on a limiter magnetically connected to the ILA is shown in [39] over a change of feeding in CD phasing.

The robustness of the simulated $V_{D C}$ poloidal structure, despite different RF designs, feeding schemes and Faraday screens, points to a possible role of the antenna box. Correlation of the poloidal structures of $V_{D C}$ and of $E_{/ /}$on the antenna frame in the input field map was noticed in [14].

For TS, 2D (radial/poloidal) maps of $V_{D C}$ were also produced using the SSWICH-FW asymptotic code [3]. In order to evidence a possible role of the Fast Wave, the code was run using either $E_{/ /}$only (dominant SW) or $E_{\text {poloidal }}$ only (dominant Fast Wave), or their combination in the vectorial input field map from RAPLICASOL. The experimental poloidal distribution of $V_{D C}$ was best reproduced with SW only. Even in the antenna vicinity the presence of the Fast Wave changed $V_{R F}$ and $V_{D C}$ magnitudes and poloidal shapes: contrary to experiments, small-scale poloidal modulations appeared in the simulations, correlated with the poloidal spacing of FS bars. It is suspected that the multi-2D approach over-estimates these poloidal modulations: it underestimates the Fast Wave radial evanescence for large poloidal wavenumbers.

\subsection{Left-right unbalance of 2-strap antennas}

The RF-induced PSI observed experimentally is weaker with two balanced straps whose toroidal phasing is

\footnotetext{
Corresponding author: laurent.colas@,cea.fr
} 
dipole $(\Delta \varphi=\pi)$ than monopole $(\Delta \varphi=0)$, (see e.g. [40]). Yet it is never totally suppressed, despite the antisymmetry of the radiated field map. In a series of TS experiments, the left-right ratio of strap voltage amplitudes was varied [36]. Over this scan, the antenna side limiter near the strap with higher voltage heated up, while the limiter at the opposite toroidal side cooled down. A similar unbalance on AUG produced opposite variations of RF currents measured at the surface of toroidally opposite antenna limiters [40]. In this experiment with $\Delta \varphi=\pi$, to minimize the collected RF current, the RF voltage imposed on the remote strap was nearly twice the RF voltage on the strap nearer to the limiter. The "optimal" voltage ratios were approximately inverse at the two toroidal sides. These trends can hardly be explained using a single physical parameter simultaneously relevant at both extremities of the same field line. They rather suggest that the toroidal distance between radiating elements and the observed walls might play a role in the RF-sheath excitation.

Within the SSWICH model these observations can be interpreted as follows. For fixed sheath widths the RF part of SSWICH is linear. For multi-port antenna excitation, it is then possible to represent the oscillating sheath voltage $V_{R F}(\mathbf{r})$ in every point $\mathbf{r}$ of the wall as a linear combination of port contributions $\left(G_{1}(\mathbf{r}), G_{2}(\mathbf{r})\right.$, $\ldots)$, weighted by the complex port voltages $\left(V_{1}, V_{2}, \ldots\right)$,

$$
V_{R F}(\mathbf{r})=V_{1} G_{1}(\mathbf{r})+V_{2} G_{2}(\mathbf{r})+\ldots
$$

For a given wall location $\mathbf{r}$, a combination of 2 port voltages always exists allowing local $V_{R F}(\mathbf{r})$ cancellation

$$
V_{2} / V_{1}(\mathbf{r})=-G_{1}(\mathbf{r}) / G_{2}(\mathbf{r})
$$

If $G_{1}(\mathbf{r})$ and $G_{2}(\mathbf{r})$ are real positive, the optimal setting is for dipole phasing (minus sign). Although a local $V_{R F}$ cancellation is always possible the optimal voltage ratio in (14) is location-dependant. Considering two toroidally opposite points $\mathbf{r}=(x, z)$ and $\mathbf{r}=(x,-z)$ on a toroidally symmetric antenna, one expects for symmetry reasons that $G_{1}(x,-z)=G_{2}(x, z)$. Relation (14) then implies that the optimal voltage ratios at the two opposite sides are inverse of each other, consistent with AUG measurements. Over left-right power unbalance, $V_{R F}(x, z)$ and $V_{R F}(x,-z)$ can vary in opposite ways, even if the two points lie on the same open magnetic field line [33]. Formula (13) also provides a general form for a scan of $\Delta \varphi$ :

$$
\begin{aligned}
& \left|V_{R F}(\mathbf{r})\right|^{2}=V_{1}^{2}\left|G_{1}(\mathbf{r})\right|^{2}+V_{2}^{2}\left|G_{2}(\mathbf{r})\right|^{2}+ \\
& \ldots 2 V_{1} V_{2} \operatorname{Re}\left[G_{1}(\mathbf{r}) G_{2}^{*}(\mathbf{r}) \exp (\mathrm{i} \Delta \varphi)\right]
\end{aligned}
$$

The detailed expressions of port contributions $G_{\mathrm{i}}(\mathbf{r})$ depend on antenna geometry and plasma parameters. Another perspective to interpret the experiments in relation with antenna geometry, is to consider the SSWICH-SW code excited by a prescribed $E_{/ /}$field map, still assuming fixed sheath widths [33]. The linearity of the RF model then implies that $V_{R F}(\mathbf{r})$ can be reexpressed formally as a linear combination of individual contributions by every emitting point in the field map.

$$
V_{R F}(\mathbf{r})=\int_{\text {aperture }} E_{/ /}\left(\mathbf{r}_{\mathbf{0}}\right) G\left(\mathbf{r}, \mathbf{r}_{\mathbf{0}}\right) \mathbf{d} \mathbf{r}_{\mathbf{0}}
$$

Expression (16) is a weighted integral of $E_{/ /}$and replaces the line integral $\tilde{V}=E_{/ /} . d l$ often used to assess RF sheaths. Symmetry arguments imply $G\left(x,-z, z_{0}\right)=G(x, z$,$z_{0}$ ) for toroidally opposite limiters. SW evanescence makes individual weights $G\left(\mathbf{r}, \mathbf{r}_{\mathbf{0}}\right)$ all the larger as the wave emission point $\mathbf{r}_{\mathbf{0}}$ is located closer to the observation point $\mathbf{r}$, both in the parallel and transverse directions. For realistic geometries and target SOL plasmas, poloidal decay occurs over a few centimetres. Typical parallel decay lengths for $G\left(\mathbf{r}, \mathbf{r}_{\mathbf{0}}\right)$ are found to be smaller than antenna parallel extension. $V_{R F}$ at antenna side limiters are therefore mainly sensitive to $E_{/ /}$ emission by metallic elements near these limiters, as suggested by experimental observations. Parallel proximity effects could also explain why sheath oscillations persist with dipole phasing, despite the parallel anti-symmetry of the radiated field map. They could finally justify reducing the RF fields induced near antenna boxes to attenuate sheath oscillations in their vicinity [40].

Paper [3] extended the previous study using fullwave calculations. In the case of Tore Supra, it showed that spatial proximity effects persist in the antenna near field in presence of both evanescent SW and Fast Waves.

\subsection{Electrical tuning of AUG 3-strap antenna}

Although the linearity makes $V_{R F}$ easier to study, deleterious RF-induced PSI arises from the DC plasma biasing. Consider one open magnetic field line of length $L_{/ /}$, exchanging DC currents with its neighbours. The sheaths at its two extremities oscillate with amplitudes $V_{R F l}$ and $V_{R F r}$. Assume that $V_{D C}$ is homogeneous along the field lines. Combining eq. (2), (3), and (11) yields

$$
\begin{aligned}
& V_{D C}=V_{f}+\ldots \\
& \frac{k T_{e}}{e} \log \left[\frac{I_{0}\left(e\left|V_{R F l}\right| / k T_{e}\right)+I_{0}\left(e\left|V_{R F r}\right| / k T_{e}\right)}{2}\right] \\
& \ldots+\frac{k T_{e}}{e} \log \left[1+\frac{L_{/ /} \operatorname{div}_{\perp} \mathbf{j}_{\perp \mathbf{D C}}}{j^{+}}\right]
\end{aligned}
$$

Where from (12) $\mathbf{j}_{\perp \mathbf{D C}}=-\sigma_{\perp \mathrm{DC}} \nabla_{\perp} V_{D C}$ in the case of Ohm's law. From relation (17) one deduces that $V_{D C}$ mitigation on the considered open field line imposes cancelling $V_{R F}$ simultaneously at its two extremities, as well as on the neighbouring field lines. Otherwise DC current flows from the high- $\left|V_{R F}\right|$ boundary to the low$\left|V_{R F}\right|$ one, without significant attenuation of $V_{D C}$.

Symmetry arguments in the previous subsection imply that the $V_{D C}$ reduction can only be partial with toroidally symmetric 2-strap antennas, over a scan of the ratio $V_{2} / V_{1}$. This was confirmed numerically on AUG using SSWICH-SW asymptotic and RAPLICASOL [14]. At fixed coupled power, the minimal $V_{D C}$ was obtained for nearly balanced antenna phased $\Delta \varphi=\pi$.

Similar symmetry considerations show that a simultaneous reduction of $V_{R F l}$ and $V_{R F r}$ is possible with 
the 3-strap AUG antenna [41] or with more straps [42]. Over a scan of $V_{2} / V_{1}$, the AUG experiment found an optimal electrical setting for which the measured tungsten (W) sputtering yield is reduced simultaneously on both toroidal sides of the AUG 3-strap antenna. This reduction was correlated with a local minimum in the RF currents collected on the frame. W production was mitigated with $\Delta \varphi=\pi$ phasing and a power ratio of the order of 2. This experiment was simulated using the SSWICH-SW asymptotic code [14], [15]. Over the parametric scan, a local minimum of the simulated $V_{D C}$ was obtained for electrical settings close to the experimental optimum. The minimal $V_{D C}$ for the 3-strap antenna was significantly smaller than the one for the 2strap antenna at the same power on the same plasma. On the RAPLICASOL input field map, the optimal setting corresponded to low values of the local $E_{/ /}$near the antenna frame, consistent with expected spatial proximity effects. Yet, complete sheath cancellation could not be achieved simultaneously everywhere over the 3-strap antenna structure: a residual RF-induced DC bias still persisted even with the optimal RF setting.

\subsection{DC plasma biasing far from the antennas}

RF-induced DC modifications of the SOL are frequently observed very far away from the active ICRF antennas, on field lines magnetically connected near the antenna limiters [43], [34], [44]. These large parallel distances (e.g. $12 \mathrm{~m}$ in [34]) contrast with the much smaller evanescence lengths for the SW invoked above to interpret the power unbalance experiments.

To explain this apparent paradox, the SSWICH-FW model proposes two possible mechanisms able to produce DC plasma biasing far from the active antenna. In the first one, propagative Fast Waves induce RF fields at remote boundaries. These $\mathrm{RF}$ far fields are subsequently rectified by "far-field" sheaths [9], [29]. An alternative mechanism was proposed in [2]: it relies on a rectification of the near RF fields in the antenna private SOL by "near-field" sheaths, and a subsequent spread of the local DC bias to remote areas by DC current transport. Using the SSWICH-FW asymptotic code, the two processes were compared numerically in [3].

In SSWICH-SW simulations of TS antennas, intense sheath oscillations were present only in the immediate vicinity of the antenna mouth [2]. In SSWICH-FW simulations using the same wall geometry, $V_{R F}$ values much larger than $k T_{e} / e$ could also be obtained on remote sheath boundaries at the LFS of the vacuum vessel, that are accessible only to the propagative Fast Wave [3]. These simulated $V_{R F}$ values were still lower than the ones obtained in the private SOL.

Over changes of the wall geometry, it was observed that the amplitude of the simulated $V_{R F}$ decreased with the parallel distance from the antenna to the observed remote sheath boundary. In particular efficient generation of $V_{R F}$ by propagative Fast Waves seems unlikely $12 \mathrm{~m}$ away from the active launcher. The simulated $V_{R F}$ did also increase with larger radial distance from the wall to the antenna. In comparison with the spatial proximity effects in the antenna near field, this result is counter-intuitive and calls for a reassessment of the "spatial proximity effects" in regions where the RF waves are propagative. One possible interpretation is that over this geometrical change, the observed sheath wall moved closer to the propagation lobe of the emitted Fast Wave.

The role of DC current transport in remote DC plasma biasing was investigated by scanning the plasma transverse DC conductivity $\sigma_{\perp D C}$ in SSWICH-FW simulations [3]. Even in presence of propagating Fast Waves, this DC plasma conductivity was found necessary to produce a $V_{D C}$ spatial distribution peaked radially at the leading edge of the side limiters, as measured experimentally [34], [43]. The amplitude and radial width of the peak was sensitive to the badly constrained value of $\sigma_{\perp \mathrm{DC}}$. A parametric scaling was proposed in [2]. The simulated $V_{D C}$ peak did propagate very far away along the magnetic field lines connected to the side limiter.

The present DC current transport model in SSWICH, despite its caveats, is yet necessary to interpret the experimental observations. TOKAM3X simulations also predict efficient parallel spread of the DC bias. The existence of DC current transport, mainly parallel to $\mathbf{B}_{\mathbf{0}}$, is also attested by several experimental observations. Biased electrodes (e.g. Langmuir probes) create DC current circuits via the plasma. DC current flows were measured experimentally from active ICRF antennas to passive objects [45], [37]. On JET, the regions of RFinduced PSI far from the antenna did move poloidally over a scan of the field line pitch angle via the safety factor $q_{95}$ [44], [39].

\subsection{RF-induced local density modifications}

Spatially inhomogeneous SOL density modifications were measured in several devices during ICRH, e.g. in [46], [47], [48]. Local RF-induced density $\mathbf{E}_{\mathbf{D C}} \times \mathbf{B}_{\mathbf{0}}$ convection is suspected in the intense DC field $\mathbf{E}_{\mathbf{D C}}=-\nabla_{\perp} V_{D C}$. The density changes in turn alter the RF wave propagation and subsequent sheath rectification. To model this process self-consistently for AUG antennas, the SSWICH-SW asymptotic code was iterated with RAPLICASOL and EMC3-EIRENE 3D SOL code for density transport [18], [19]. In EMC3, $V_{D C}$ was assumed homogeneous along the open field lines. The obtained self-consistent density patterns were compared with local measurements from reflectometers embedded in the AUG 3-strap antenna, showing reasonable agreement.

\section{Conclusions and outlook.}

Within the reported project, important upgrades were realized in the SSWICH code, coupling RF wave propagation and DC SOL biasing via sheath boundary conditions (SBCs). Full-wave RF wave propagation was implemented in 2D (radial/toroidal) geometry, together 
with PMLs and magnetized sheaths at shaped walls, for tokamaks and the RF-sheath testbed ALINE.

Even after these upgrades, present « antenna-scale » models remain relatively simple. The geometry is simpler than a real tokamak vessel and focuses on the antenna vicinity. Most tokamak simulations accounted for the Slow Wave (SW) only. The multi-2D approach followed, although well-suited for the SW, appears questionable to reproduce the Fast Wave evanescence for small-scale poloidal modulations in the input RF field maps. The implemented SBCs are simpler than more detailed fluid or PIC models of RF sheaths. Most tokamak simulations used asymptotic RF-SBCs that are even simpler. DC current transport relies on a phenomenological Ohm's law that cannot account for the turbulent DC current conduction likely at play. In the prospect of quantitative prediction, further improvement is needed from more basic research into RF-sheath physics, SOL turbulence and applied mathematics.

In its simplest form the SSWICH code gets now widely used in Europe. Despite its relative simplicity, it was already able to reproduce qualitative experimental observations: the poloidal distributions of RF-induced near-field PSI over many antenna configurations; the radial position of $V_{D C}$ peaks; left-right asymmetries upon strap power unbalance; electrical tuning of various antennas; spread of DC bias over long parallel distances and DC current flows. Earlier models, e.g. the oscillating double probe analogy with line integral $\tilde{V}=E_{/ /} \cdot d l$, failed to interpret some of these observations. From these studies, some key physical ingredients have emerged as essential to reproduce the measurement results: accurate input RF field maps from 3D antenna codes; spatial proximity effects in the near field due to SW and possibly fast wave evanescence; parallel and transverse DC current transport.

Detailed comparisons with local measurements, especially 2D mappings, were essential for assessment. Of particular interest for future investigation would be local measurements in the antenna private SOL, where the rectification is predicted most intense. Measurements might be easier in RF-sheath testbeds, motivating more extensive simulation of these devices.

Presently SSWICH-SW can be used to assess parametric dependences and compare antenna designs. Spatial proximity effects provide guidelines for future antenna optimization. Yet, aside from the numerous approximations made, sensitivity of the results to loosely constrained parameters prevents good quantitative prediction. The amplitude and radial width of $V_{D C}$ peaks were found sensitive to uncertainties in the measured profiles, private SOL poorly accessible to diagnostics [20], RF-induced local SOL modifications [15], and transverse DC conductivity $\sigma_{\perp \mathrm{DC}}[2]$. Predicting "far field sheaths" is probably even more critical.

Acknowledgements. This work has been carried out within the framework of the EUROfusion Consortium and has received funding from the European research and training programme under grant agreement $\mathrm{N}^{\circ} 633053$. The views and opinions expressed herein do not necessarily reflect those of the European Commission.

\section{References}

1. L. Colas et al., Phys. Plasmas 19092505 (2012)

2. J. Jacquot et al. Phys. Plasmas 21, 061509 (2014)

3. L. Lu, et al., "Non-linear RF wave-sheath interaction in magnetized plasma edge: the role of the fast wave", ECA Vol. 40A, P2.068, http://ocs.ciemat.es/EPS2016PAP/pdf/P2.068.pdf, to be submitted to $P P C F$

4. D.A. D'Ippolito \& J.R. Myra, Physics of Plasmas 13 102508 (2006)

5. H. Kohno et al. Computer Physics Communications 183 (2012) p. 2116

6. J. R. Myra and D. A. D'Ippolito, Phys. Plasmas 22, 062507 (2015)

7. J.R. Myra, Physics of Plasmas 24, 072507 (2017)

8. D. Van Eester and K. Crombé, Plasma Physics and Controlled Fusion 55 (2013) 055001

9. D. A. D'Ippolito et al., Physics of Plasmas 15, 102501 (2008)

10. D. Van Eester and K. Crombé, Phys. Plasmas 22, 122505 (2015)

11. T.G. Jenkins and D.N. Smithe, Plasma Sources Sci. Technol. 24 (2015) 015020

12. T.H. Stix, "Waves in plasmas", 1992, AIP Press

13. J. Jacquot et al., AIP Conference Proceedings 1689, 050008-1 050008-4 (2015)

14. J. Jacquot et al., this conference poster B-39

15. Tierens W. et al, Nucl. Fusion 57 (2017) 116034

16. W. Tierens, Poster A-25, this conference

17. J. Jacquot et al., Plasma Phys. Control. Fusion 55 (2013) 115004

18. Zhang. W et al., Nucl. Fusion 57 (2017) 116048

19. W. Zhang et al., Inv-07, this conference

20. L. Lu et al., PPCF 58 (2016) 055001

21. K.G. Budden, "Radio Waves in the Ionosphere", Cambridge University Press 1961

22. M. Campos-Pinto and B. Després, "Constructive formulations of resonant Maxwell's equations", to appear in Siam Journal Math. Anal. 2017, https://hal.archives-ouvertes.fr/hal01278860/document.

23. B. Després et al., poster $\mathrm{C}-32$, this conference

24. Crombé, K. et al., AIP Conference Proceedings, 1689, 030006 (2015)

25. E. Faudot et al., Review of Scientific Instruments, $\mathbf{8 6}$ 063502 (2015).

26. E. Faudot et al. poster A-33, this conference

27. P. Tamain, et al., Nucl. Materials and Energy (2017), http://dx.doi.org/10.1016/j.nme.2016.12.022

28. T.G. Jenkins et al., poster B-30, this conf.

29. H. Kohno et al., Phys. Plasmas 22, 072504 (2015), ibid. 23 (2016) 089901. 
30. L. Colas et al., Proc. $21^{\text {st }}$ IAEA FEC conference, St Petersburg (Russia) 2014, TH/P6-9

31. A. Křivská et al., poster C-44, this conference

32. D. Milanesio et al., Nuclear Fusion 49 (2009) 115019

33. L. Colas et al., Plasma Phys. Control. Fusion 59 (2017) 025014

34. M. Kubič et al., Journal of Nuclear Materials 438 (2013) S509-S512

35. L. Colas et al. AIP conf. proc. 1580 p.259 (2014)

36. L. Colas et al., JNM 4382013 S330-3

37. V.V. Bobkov et al. Nuclear Fusion 50 (2010) 035004

38. B. VanCompernolle et al., poster C-26, this conf.

39. C.C. Klepper et al., poster A-12, this conference

40. V. Bobkov et al., AIP Conf. Proc. 1689, 030004-1 030004-8 (2015)

41. V. Bobkov et al., Plasma Phys. Control. Fusion 59 (2017) 014022

42. S.J. Wukitch et al., Inv-08 this conference

43. Cziegler I. et al., Plasma Physics and Controlled Fusion 54 (2012) 105019

44. C.C. Klepper et al. Journal of Nuclear Materials 438 (2013) S594

45. R. Van Nieuwenhove and G. Van Oost, PPCF 34 (4), pp.525-532 (1992)

46. Bécoulet M. et al., Physics of Plasmas 9 (2002) pp. 2619-32

47. Lau C. et al., PPCF 55 (2013) 095003

48. Colas L. et al., (2015) JNM 463 pp.735-8 